\section{Buchrezension zu: Was ist Leben?}

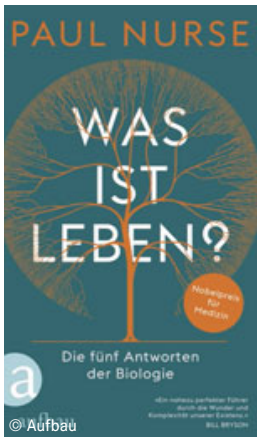

Was ist Leben?

Die fünf Antworten der

Biologie

Paul Nurse

184 S., Aufbau, 2021. HC mit Schutzumschlag, 20,00 €.

ISBN: 9783351038885

DOI: $10.1007 / \mathrm{s} 12268-022-1703-\mathrm{Z}$ (C) Der Autor 2022

Papa, was ist das? Eine Amsel. Papa, ist die tot? Nee schau, die bewegt sich. Die lebt. Papa, was heißt das, die lebt? Tja, was ist Leben? Bei dieser einfachen Dreiwortfrage geraten auch erfahrene Biologen gerne ins Stocken. Der Nobelpreis-gekrönte Genetiker und Zellbiologe Paul Nurse widmet sich dieser grundlegenden Frage auf knapp 200 Seiten, die beim Lesen wie im Flug vergehen. Nurse schöpft aus einem großen Fundus jahrzehntelanger Erfahrung und führt eine leichte Feder mit sicherer Hand, was das Buch trotz höchst anspruchsvoller Materie zu einem Lesevergnügen für ein breites Zielpublikum macht. Obgleich der schnörkellosen Erzählweise kommt „Was ist Leben“ notgedrungen nicht ohne eine Vielzahl an Fachbegriffen aus, was interessierten Lai:innen meines Erachtens aber nicht den Zugang verwehrt.

Das Buch steht in der Tradition gleichnamiger Werke des Physikers Erwin Schrödinger oder des Evolutionsbiologen J. B. S. Haldane, die Mitte des letzten Jahrhunderts dieselbe Frage aus ihrer jeweiligen Perspektive zu beleuchten suchten. Paul Nurse wählt einen umfassenden Ansatz und entführt die Leser:innen auf eine spannende Reise durch die Welt der Biologie. Er bereist Länder unterschiedlicher Fachdisziplinen, gewährt Einblicke in ihre Geschichte, vermisst Ideenräume und vermittelt zentrale Konzepte und Einsichten. Klug gewählte Analogien aus der Lebenswirklichkeit der Leser:innen sowie persönliche Erfahrungen und Anekdoten aus der Forschungswirklichkeit von Kollegen machen die oft komplexe Materie gut verständlich und erlebbar. Die unmittelbar in der Forschung des Autors verankerten Erkenntnisse der Zellbiologie und Genregulation sind besonders lesenswert und bieten auch dem biologisch vorgeschulten Publikum einen reichhaltigen Fundus an gut sortiertem Wissen. Alles in allem entsteht beim Lesen, fast nebenbei, fachlich fundiertes Grundwissen, das am Ende des Buchs für die Synthese und Einordung in aktuelle gesellschaftliche Debatten zum Tragen kommt.

Wer eine Dreiwortantwort auf die Frage nach dem Wesen des Lebens sucht, ist mit „Was ist Leben?" falsch beraten. Wer hingegen neugierig ist, mehr über eine hochdynamische wissenschaftliche Disziplin zu erfahren, die tief bis in alle unsere Lebensbereiche vordringt, dem ist Paul Nurses Versuch, das Wesen des Lebens zu ergründen, wärmstens zu empfehlen.

Jochen Wolf, LMU München,

j.wolf@biologie.uni-muenchen.de

Diese Rezension erscheint Open Access. *
Funding note: Open Access funding enabled and organized by Projekt DEAL. Open Access: Dieser Artikel wird unter der Creative Commons Namensnennung 4.0 International Lizenz veröffentlicht, welche die Nutzung Vervielfältigung, Bearbeitung, Verbreitung und Wiedergabe in jeglichem Medium und Format erlaubt, sofern Sie den/die ursprünglichen Autor(en) und die Quelle ordnungsgemäß nennen, einen Link zur Creative Commons Lizenz beifügen und angeben, ob Änderungen vorgenommen wurden. Die in diesem Artikel enthaltenen Bilder und sonstiges Drittmaterial unterliegen ebenfalls der genannten Creative Commons Lizenz, sofern sich aus der Abbildungslegende nichts anderes ergibt. Sofern das betreffende Material nicht unter der genannten Creative Commons Lizenz steht und die betreffende Handlung nicht nach gesetzlichen Vorschriften erlaubt ist, ist für die oben aufgeführten Weiterverwendungen des Materials die Einwilligung des jeweiligen Rechteinhabers einzuholen. Weitere Details zur Lizenz entnehmen Sie bitte der Lizenzinformation auf

http://creativecommons.org/licenses/ by $/ 4.0 /$ deed.de. 